\title{
Dosimetric predictors of nausea and vomiting: an exploratory analysis of a prospective phase I/II trial with neoadjuvant accelerated short-course radiotherapy and capecitabine for resectable pancreatic cancer
}

\author{
Yolanda D. Tseng • Jennifer Y. Wo • Marek Ancukiewicz • \\ Judith Adams • Nicolas Depauw • Harvey J. Mamon • \\ Theodore S. Hong
}

Received: 29 April 2013 / Accepted: 12 August 2013 / Published online: 22 August 2013

(C) Springer-Verlag Berlin Heidelberg 2013

\begin{abstract}
Objective We performed an exploratory analysis to identify dose-volume parameters that may predict treatment-induced nausea and vomiting among patients receiving hypofractionated radiotherapy to the pancreas.

Methods A retrospective analysis was performed of 47 patients with localized, resectable pancreatic adenocarcinoma who were prospectively treated on a phase I/II protocol between 2007 and 2010. Patients received proton beam radiotherapy (5 GyE x 5) concurrent with capecitabine $\left(825 \mathrm{mg} / \mathrm{m}^{2}\right.$ twice daily) over 1 $(n=41)$ or 2 weeks $(n=6)$. Kendall's $\tau$ correlation coefficients were calculated between dosimetric parameters of the planning treatment volume, stomach, duodenum, liver, small and large bowel, and physician-reported nausea or vomiting. Recursive partitioning analysis was performed to identify the best binary split of dosimetric predictors for nausea or vomiting.
\end{abstract}

This work was presented in part at the 54th meeting of the American Society for Therapeutic Radiology and Oncology, Boston, MA, October 28-31, 2012.

Y. D. Tseng $(\bowtie)$

Harvard Radiation Oncology Program, 75 Francis Street, Boston,

MA 02115, USA

e-mail: ydtseng@partners.org

J. Y. Wo $\cdot$ M. Ancukiewicz $\cdot$ J. Adams $\cdot$ N. Depauw $\cdot$ T. S. Hong Department of Radiation Oncology, Massachusetts General Hospital, Harvard Medical School, Boston, MA, USA

\section{H. J. Mamon}

Department of Radiation Oncology, Dana-Farber Cancer Institute/ Brigham and Women's Hospital, Harvard Medical School, Boston, MA, USA

N. Depauw

Centre for Medical Radiation Physics, University of Wollongong, Wollongong, NSW 2500, Australia
Results Hypofractionated radiation to the pancreas was welltolerated with 51 and $4 \%$ of patients experiencing grades 1 and 2 nausea or vomiting, respectively. Mean dose to the stomach ( $\mathrm{D}_{\text {mean }}, \tau=0.33 ; p=0.008$ ) and volume of stomach that received 3 GyE (V3, $\tau=0.34 ; p=0.005$ ), 5 GyE (V5, $\tau=0.35$; $p=0.005), 10 \mathrm{GyE}(\mathrm{V} 10, \tau=0.31 ; p=0.01)$, and $15 \mathrm{GyE}$ or higher $(\mathrm{V} 15, \tau=0.27 ; p=0.03)$ were all directly associated with nausea or vomiting during treatment. Stomach V10 $\geq 11.5 \%$ was the best predictor of nausea or vomiting: $20 \%$ versus $72 \%$ patients experienced nausea or vomiting with $\mathrm{V} 10<11.5$ versus $\geq 11.5 \%$.

Conclusion Low-dose hypofractionated radiation to the stomach is associated with nausea or vomiting during and immediately after radiotherapy. While these findings require validation, they may help identify patients who could benefit from prophylactic antiemetic medications during radiotherapy.

Keywords Radiotherapy $\cdot$ Nausea $\cdot$ Capecitabine $\cdot$

Dosimetry

\section{Introduction}

Radiation-induced nausea and vomiting is a well-recognized but under-studied acute toxicity of radiotherapy. Young age, female gender, and minimal consumption of alcohol have been associated with an increased risk of nausea and vomiting during chemotherapy [1], while concomitant chemotherapy and prior vomiting induced by chemotherapy have been associated with an increased risk of nausea and vomiting during radiotherapy $[2,3]$. Little is known, however, about radiation treatmentrelated risk factors. Large treatment field size $\left(>400 \mathrm{~cm}^{2}\right)$ and radiation to the upper abdomen have been associated with an increased risk of nausea, vomiting, or both [2,3], but the organs 
and dose-volume parameters that mediate the increased nausea and vomiting risk are less well-known.

Radiation-induced emesis has previously been observed to be higher after mid- and upper-hemibody irradiation compared with lower-hemibody irradiation $[4,5]$, suggesting that the critical organs responsible for treatment-induced nausea and vomiting might reside in the upper abdomen. One hypothesis is that damage to normal organ structures, in particular the enterochromaffin cells of the gastrointestinal (GI) mucosa, releases serotonin and subsequently initiates the emetogenic response through the binding of 5-hydroxytryptamine receptors and downstream activation of the chemoreceptor trigger zone in the brain [1]. Identification of organs at risk (OAR) that may mediate treatment-induced nausea and vomiting would allow radiation oncologists to minimize dose to these structures, to identify high-risk patients, or both.

With a quick treatment course and favorable toxicity profile, hypofractionated radiotherapy to the pancreas increasingly has been used to treat locally advanced [6] or unresectable pancreatic cancer $[7,8]$. However, the dosimetric parameters that mediate acute toxicity such as nausea and vomiting are not well-defined within this growing patient population. We therefore performed this exploratory analysis to retrospectively identify dosimetric parameters that predict the risk of any nausea, vomiting, or both among patients prospectively treated on a phase I/II study of neoadjuvant capecitabine and hypofractionated proton radiotherapy to the pancreas for resectable pancreatic adenocarcinoma.

\section{Methods}

\section{Patient population}

The study cohort comprised of 50 patients treated prospectively from June 2007 to December 2010 on a phase I/II study of neoadjuvant accelerated short-course radiotherapy and capecitabine. Prior to enrollment, all patients gave informed consent to participate. Three patients were excluded given that they received 30 cobalt gray equivalents (GyE) in ten fractions, while subsequent patients all received 25 GyE in five fractions. This left 47 patients eligible for analysis.

Details of this study have been previously reported [9]. Briefly, this trial included 50 patients with biopsy-proven adenocarcinoma of the pancreatic head or neck amenable to a pancreaticoduodenectomy. Resectability was based on a pancreatic protocol computed tomography (CT) scan and no evidence of metastatic disease on CT of the torso and diagnostic laparoscopy. All patients were required to have an Eastern Cooperative Oncology Group performance status of 0 or 1. Patients were excluded if they had received therapy for pancreatic cancer, radiotherapy, or chemotherapy in the last 5 years.
Radiotherapy, chemotherapy, and surgical treatment

All patients underwent four-dimensional (4D) simulation with intravenous and oral contrast $(450 \mathrm{~mL})$, and the gross tumor volume (GTV) was contoured with the aid of a pancreatic protocol CT scan. The clinical target volume included the GTV with a 1-cm margin and moderate elective nodal coverage of the celiac axis, porta hepatis, superior mesenteric artery, and pancreaticoduodenal region as defined by the inner-third of the duodenum. Planning target expansion was customized and depended on the estimated setup variation and organ motion from the 4D simulation CT scan. Radiotherapy was delivered using protons generated from a cyclotron at Massachusetts General Hospital. Treatment plans generally comprised three fields, with two fields being treated per day (Fig. 1). Total dose was prescribed to the $95 \%$ isodose line. Based on our clinical experience, some patients with mild nausea have found taking small amounts of food or liquid to be therapeutic. Therefore, we had no firm restrictions regarding oral intake prior to each treatment.

During phase I, proton beam radiotherapy was delivered on a progressively accelerated schedule with an overall treatment time decreasing from 2 to 1 week. The first three patients received a total of $30 \mathrm{GyE}$ over ten treatments (12 days) and were excluded from this analysis. Thereafter, each group of three patients was treated with $25 \mathrm{GyE}$ in five treatments spread over a progressively shorter period: 11 days ( $n=3), 9$ days $(n=3)$, and finally 5 days $(n=41)$. Normal tissue dose constraints were followed for liver, kidney, spinal cord, and stomach [9].

All radiotherapy was given concurrently with capecitabine at a fixed dose of $1,650 \mathrm{mg} / \mathrm{m}^{2}$ by mouth divided twice daily. Capecitabine was given Monday through Friday for 2 weeks or a total of 10 days. All 47 patients were counseled to use ondansetron ( $8 \mathrm{mg}$ by mouth) 30 to $60 \mathrm{~min}$ before therapy for prophylaxis. Patients were also initiated on a proton pump inhibitor if they were not already taking one. After completion

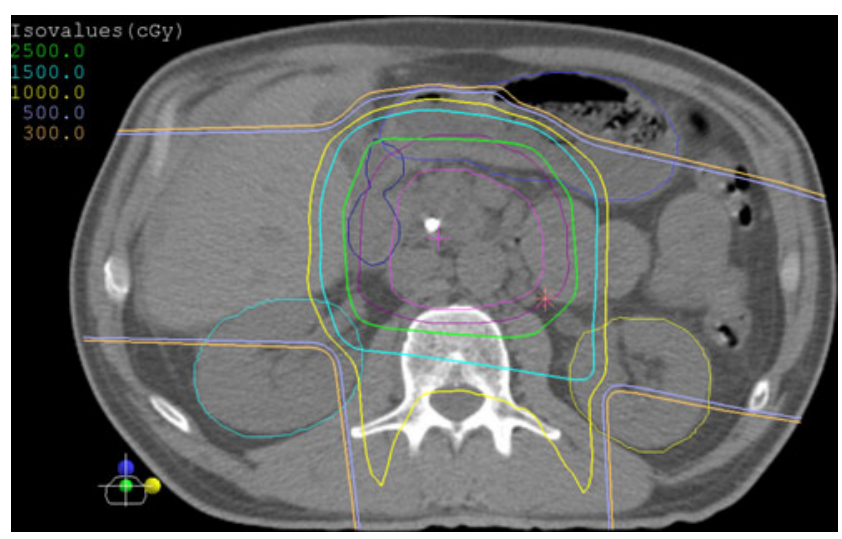

Fig. 1 Axial slice of a proton treatment plan volume and isodose distribution. Primary tumor is outlined in pink 
of chemoradiation, patients underwent surgery 1 to 6 weeks after the last dose of capecitabine.

\section{Clinical and DVH parameters}

Both clinical and dosimetric variables were retrospectively extracted for this exploratory analysis with approval from the Dana-Farber/Harvard Cancer Center institutional review board. Clinical parameters of interest included patient's age, gender, BMI, alcohol intake, presence of diabetes, history of abdominal surgeries, and baseline use of any antiemetic medication at the time of radiation consultation. An experienced anatomist and dosimetrist (JA) contoured the liver, stomach, duodenum, and small and large bowel as potential OARs with the treating physicians (JYW, TSH). Size of the planning target volume (PTV) and percentage of OAR volume receiving $3,5,10,15,20$, and $25 \mathrm{GyE}$, in addition to the maximum and mean GyE, were extracted from the patient's treated proton plan (Fig. 2; XiO planning software, Elekta, Sweden).

\section{Assessment of nausea and vomiting}

The primary endpoint of this study was treatment-related nausea, vomiting, or both during and up to 1 week after radiotherapy completion. A single investigator (YDT) reviewed each treating physician's on-treatment and 1-week follow-up notes and retrospectively graded physician-reported nausea and vomiting using the NCI common terminology criteria for adverse events (v3.0). The highest toxicity grade among the week 1, week 2, and 1week follow-up visits was recorded.
We also collected other, indirect measurements of nausea and vomiting. As part of the prospective phase I/II protocol, enrolled patients completed a daily medication diary throughout their 2-week radiotherapy and capecitabine course and recorded the number and type $(8 \mathrm{mg}$ ondansetron, $10 \mathrm{mg}$ prochlorperazine) of oral antiemetic medication used. In addition to the number of antiemetic pills used, we examined whether rescue medication was required, defined as more than one antiemetic pill taken in a single day. The 45 patients who returned their diaries were included in this analysis. Lastly, we calculated both absolute and percent weight change over treatment for 46 patients whose weights were recorded at the start of radiotherapy and on or within 1 week of radiotherapy completion.

\section{Statistical methods}

Patient characteristics and dose-volume parameters of normal organ structures were summarized by descriptive statistics. Kendall's tau correlation coefficients were calculated between dosimetric parameters of the PTV, stomach, duodenum, liver, small and large bowel, and treatment-related nausea, vomiting, or both during and up to 1 week after radiotherapy completion. Differences in baseline patient characteristics among patients who experienced nausea or vomiting and those who did not were assessed using the chi-square statistic for categorical variables and a $t$ test for continuous variables. Differences in dosimetric parameters between patients who did and did not experience nausea and vomiting were evaluated using the exact Mann-Whitney test. We used recursive
Fig. 2 Corresponding dosevolume histogram (DVH) of the treatment volume shown in Fig. 1. The clinical treatment volume (CTV), planning treatment volume (PTV), liver, stomach, duodenum, small bowel, and large bowel DVH are shown

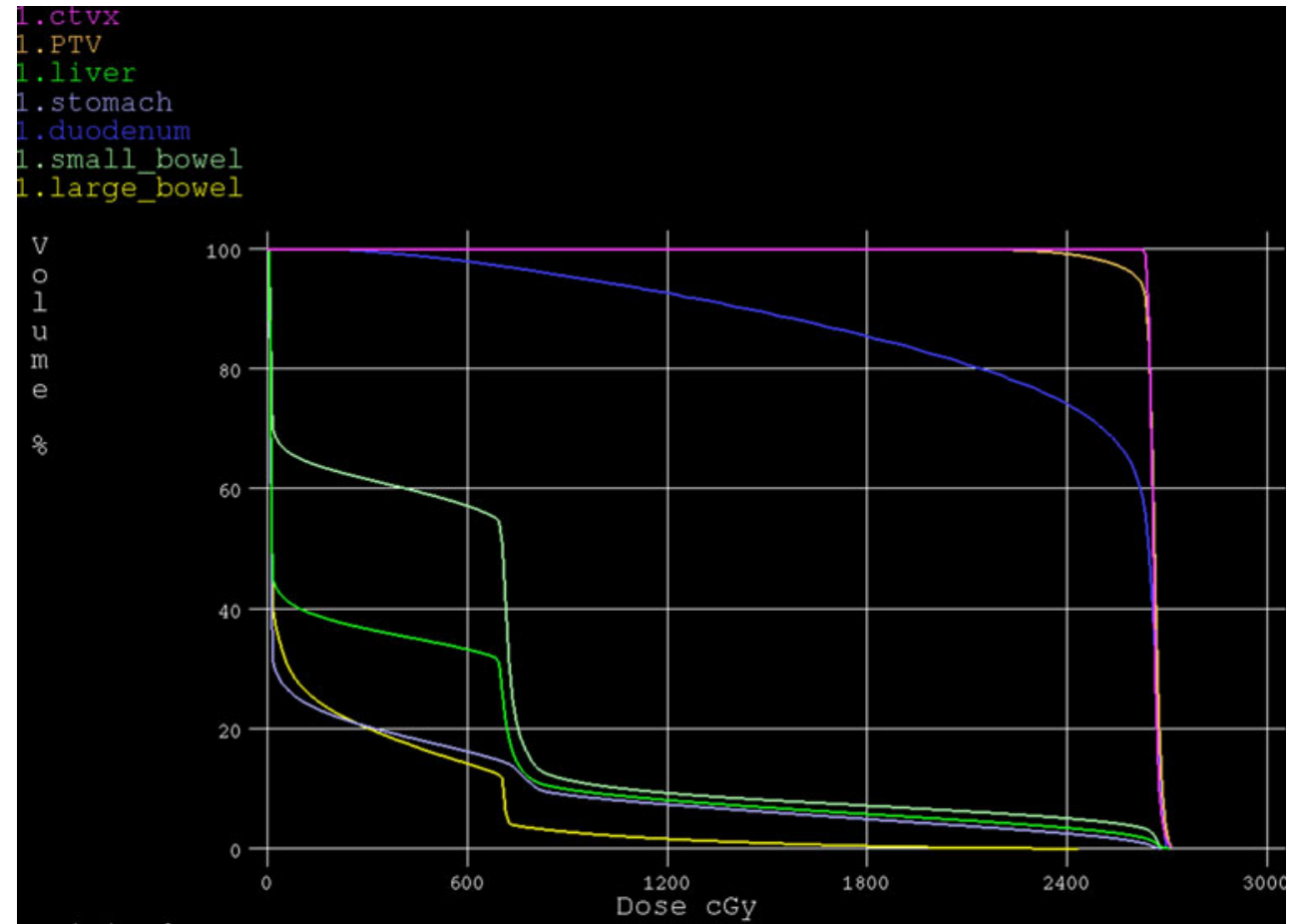


partitioning analysis (RPA) to identify dosimetric parameters and binary splits that best predicted the risk of nausea or vomiting. Given the exploratory nature of our analysis, we did not adjust for multiple statistical tests; rather, each parameter was of separate interest. $P$ values less than 0.05 were considered significant, and all tests were two-sided.

\section{Results}

Patient characteristics, antiemetic medication use, and treatment tolerance

Forty-seven patients were included in this cohort: $53 \%$ were female and the mean age was 66.0 years (standard deviation [SD], 9.6) (Table 1). Only five patients (11\%) were on an antiemetic medication (most commonly a benzodiazepine) at the time of radiation consultation. No significant differences in baseline patient and medical characteristics were detected between patients who experienced nausea, vomiting, or both during treatment and patients who did not. Throughout the 2week treatment course, patients took on average 13.1 antiemetic pills (SD, 9.6) for prophylaxis, rescue, or both. While daily ondansetron was recommended for prophylaxis, this was not consistently taken by all patients and on all treatment days. Sixty-four percent required rescue medication over an average of 8.4 days (SD, 3.1). The mean time to rescue medication use was 3.6 days (SD, 3.3) (Table 2). Although nonsignificant, a higher proportion of patients who experienced nausea or vomiting during treatment required rescue medications compared to those who did not: 73 versus $55 \%(p=0.34)$.

Treatment was overall well-tolerated with 51 and $4 \%$ of patients experiencing physician-reported grades 1 and 2 nausea and vomiting, respectively; no patients experienced grade 3 or 4 nausea and vomiting. Patients lost on average $1.63 \mathrm{lb}$ (SD, 3.24 ) or $0.9 \%(\mathrm{SD}, 1.8 \%)$ of their initial weight (Table 2$)$.

Dosimetric predictors of physician-reported nausea and vomiting

Mean dose to the stomach (Kendall's $\tau$ coefficient, 0.33; $p=0.008$ ), volume of stomach that received $3 \mathrm{GyE}$ or greater (V3; $\tau=0.34 ; p=0.005$ ), $5 \mathrm{GyE}$ or greater (V5; $\tau=0.35 ; p=$ $0.005), 10 \mathrm{GyE}$ or greater (V10; $\tau=0.31 ; p=0.01$ ), and $15 \mathrm{GyE}$ or greater $(\mathrm{V} 15 ; \tau=0.27 ; p=0.03)$ were all significantly correlated with the presence of nausea, vomiting, or both during treatment (Table 3). The mean dose to the stomach was significantly higher among patients who developed nausea, vomiting, or both compared to those who did not $\left(\mathrm{D}_{\text {mean }} 4.6\right.$ GyE versus $3.4 \mathrm{GyE} ; p=0.007)$. PTV, irradiated volume of liver, duodenum, small bowel, and large bowel were not significantly correlated with presence of nausea, vomiting, or both during treatment. On RPA, the dosimetric parameter and split that optimally predicted for occurrence of nausea or vomiting was stomach $\mathrm{V} 10<11.5 \%$ and $\geq 11.5 \%$. Among 15 patients with a stomach $\mathrm{V} 10<11.5 \%, 20 \%$ experienced nausea or vomiting versus $72 \%$ of 32 patients with a stomach V10 $\geq 11.5 \%$ had nausea or vomiting.

\section{Discussion}

Prior studies have confirmed that radiotherapy to the upper abdomen concurrent with capecitabine is well-tolerated with an acceptable acute toxicity profile [10-12]. Most treatmentassociated nausea and vomiting is limited to grade 1 or 2 severity, with only $3 \%$ and $5 \%$ of patients in a retrospective study from MD Anderson experiencing physician-reported

Table 1 Patient characteristics stratified by presence of nausea, vomiting, or both during radiotherapy and capecitabine treatment

\begin{tabular}{lllc}
\hline & $\begin{array}{l}\text { No nausea and } \\
\text { vomiting }(n=21)\end{array}$ & $\begin{array}{l}\text { Nausea, vomiting, } \\
\text { or both present }(n=26)\end{array}$ & All $(n=47)$ \\
\hline Age at RT consultation, mean years (SD) & $66.5(8.9)$ & $65.6(10.3)$ & $66.0(9.6)$ \\
Female gender & $10 / 21(47.6 \%)$ & $15 / 26(57.7 \%)$ & $25 / 47(53.2 \%)$ \\
BMI, mean (SD) & $26.6(4.3)$ & $27.4(5.4)$ & $27.0(4.9)$ \\
Alcohol consumed & $14 / 21(66.7 \%)$ & $22 / 26(84.6 \%)$ & $36 / 47(76.6 \%)$ \\
$\quad$ None or social & $3 / 21(14.3 \%)$ & $2 / 26(7.7 \%)$ & $5 / 47(10.6 \%)$ \\
$\quad$ History of alcohol abuse & $2 / 21(9.5 \%)$ & $1 / 26(3.8 \%)$ & $3 / 47(6.4 \%)$ \\
$\quad 1-2$ units/day & $2 / 21(9.5 \%)$ & $1 / 26(3.8 \%)$ & $3 / 47(6.4 \%)$ \\
$\quad>2$ units/day & $1 / 21(4.8 \%)$ & $4 / 26(15.4 \%)$ & $5 / 47(10.6 \%)$ \\
Use of antiemetics at baseline & & & 0.54 \\
Comorbidities & $4 / 21(19.0 \%)$ & $4 / 26(15.4 \%)$ & $8 / 47(17.0 \%)$ \\
$\quad$ Diabetes & $10 / 21(47.6 \%)$ & $13 / 26(50.0 \%)$ & $23 / 47(48.9 \%)$ \\
History of abdominal surgery & & & 1.00 \\
\hline
\end{tabular}

$S D$ standard deviation 
Table 2 Mean weight change and use of antiemetic medications stratified by presence or absence of physician-reported nausea and vomiting. Average changes in weight were calculated for 46 patients with available data; the remaining summary statistics on medication use were based on 45 patients who returned completed medication diaries

\begin{tabular}{|c|c|c|c|c|}
\hline & $\begin{array}{l}\text { No nausea } \\
\text { and vomiting }\end{array}$ & $\begin{array}{l}\text { Nausea, vomiting, } \\
\text { or both present }\end{array}$ & All & $P$ value \\
\hline Weight difference, mean pounds (SD) & $-1.11(2.76)$ & $-2.02(3.57)$ & $-1.63(3.24)$ & 0.32 \\
\hline Percent weight change (SD) & $-0.8 \%(1.7)$ & $-1.1 \%(1.9)$ & $-0.9 \%(1.8)$ & 0.55 \\
\hline Number of prochlorperazine pills used (SD) & $5.6(9.7)$ & $5.2(6.0)$ & $5.4(7.9)$ & 0.44 \\
\hline Number of ondansetron pills used (SD) & $7.4(3.9)$ & $8.2(5.7)$ & $7.8(4.9)$ & 0.58 \\
\hline Number antiemetic pills (prochlorperazine + ondansetron) used (SD) & $13.4(10.3)$ & $12.9(9.3)$ & $13.1(9.6)$ & 0.97 \\
\hline Need for rescue medication & $11 / 20(55.0 \%)$ & $16 / 22(72.7 \%)$ & $27 / 42(64.3 \%)$ & 0.34 \\
\hline Days until first rescue medication used (SD) & $2.5(2.3)$ & $4.3(3.7)$ & $3.6(3.3)$ & 0.16 \\
\hline Number of days requiring rescue medication (SD) & $8.2(3.6)$ & $8.8(2.9)$ & $8.4(3.1)$ & 0.70 \\
\hline
\end{tabular}

$S D$ standard deviation

grade 3 nausea and vomiting, respectively [11]. In our study, $55 \%$ of patients experienced grade 1 or 2 nausea and vomiting and no patients suffered grade 3 or higher nausea and vomiting. These favorable rates of nausea and vomiting may in part be secondary to aggressive treatment with prophylactic antiemetic medications. Our rates of nausea and vomiting are consistent with the $53 \%$ rate of grade 1 or 2 nausea reported by Ben-Josef and colleagues [10]. In this retrospective study from Wayne State, patients with pancreatic adenocarcinoma received a similar dose of capecitabine $\left(800 \mathrm{mg} / \mathrm{m}^{2}\right.$ twice daily) with conventionally fractionated, intensity-modulated radiotherapy (IMRT, 45-55 Gy).

Despite nausea and vomiting being the common side effects of radiotherapy to the pancreas, the mechanisms of toxicity are not well understood. While patient-related risk factors have been identified among chemotherapy [13] and radiotherapy-based studies [2], only a few studies have identified OARs and investigated dosimetric-risk factors $[2,3,14$, 15]. In our exploratory analysis, mean $\left(D_{\text {mean }}\right)$ and low-dose, hypofractionated radiotherapy to the stomach (V3, V5, V10, V15) were the only significant dosimetric variables correlated with the presence of nausea, vomiting, or both during concurrent radiotherapy and capecitabine treatment, and stomach $\mathrm{V} 10 \geq 11.5 \%$ was the best predictor of nausea or vomiting.

Our results are consistent with the findings of prior studies and clinical, anecdotal experience; in a prospective, multicenter study with 934 consecutive patients receiving radiotherapy, upper abdomen radiation was a significant risk factor for radiation-induced nausea, vomiting, or both [3]. The stomach has been identified as an OAR for acute gastrointestinal (GI) toxicity. In this contemporary analysis from the Kyoto University Graduate School of Medicine, however, high rather than low dose to the stomach, and in particular V50 $\geq 16 \mathrm{~cm}^{3}$, was the best predictor of grade 2 or higher acute GI toxicity ( $9 \%$ if $<16$ versus $61 \%$ if $\geq 16 \mathrm{~cm}^{3} ; p=0.001$ ) among patients with locally advanced pancreatic cancer [15]. Several factors may help explain our discrepant dosimetric findings. Compared to our study, patients in this study had more advanced pancreatic disease, received gemcitabine instead of capecitabine chemotherapy, and were treated with conventionally fractionated radiotherapy (54 Gy in 30 fractions). Moreover, while we used the presence of any nausea, vomiting, or both during treatment as our endpoint, this study examined grade 2 or higher nausea, vomiting, anorexia, and mucositis. While such differences prevent direct comparison, they highlight the stomach as a potential mediator of acute radiation-induced GI toxicity.

Given the stomach's proximity to the target volume, minimizing dose to the stomach can be challenging. There is no clear, optimal treatment planning approach. Two studies have compared dosimetry between three-dimensional (3D) conformal radiotherapy (CRT) with protons (2-3 fields) and photon-based IMRT. Both studies evaluated treatment to a pancreatic primary and suggested that while protons are superior to IMRT based on the volume of stomach that receives low radiation doses [16, 17], protons deliver high radiation doses to a larger volume of stomach than IMRT photon-based planning [16]. While coverage of target volume may be inferior with photon-based 3D CRT compared with IMRT or proton-based 3D CRT [17], it is unknown how photon-based 3D CRT compares with IMRT or protons with respect to stomach dose and whether this is associated with significant differences in rates of treatment-induced nausea or vomiting. In one study of patients treated for pancreatic or ampullary cancers, IMRT was associated with lower rates of grade 3 and 4 nausea and vomiting compared with 3D treatment planning ( 0 versus $11 \%, p=0.024)$ [18]. However, no dosimetric comparisons were made between IMRT and 3D plans; therefore, it is unclear what mediated this improvement. In our study using proton radiotherapy, no grade 3 or 4 nausea and vomiting was observed and it is unclear whether IMRT would improve grade 1 and 2 nausea and vomiting.

We did not detect significant differences in baseline patient characteristics among those who experienced nausea, vomiting, 
Table 3 Summary of dose-volume histogram and Kendall's correlation coefficients between dose-volume parameters and physician-reported nausea and vomiting

\begin{tabular}{|c|c|c|c|}
\hline & $\begin{array}{l}\text { Mean volume }(\%) \\
\text { or dose }(\mathrm{GyE}) \text { with } \\
\mathrm{SD}(n=47)\end{array}$ & $\begin{array}{l}\text { Kendall's correlation } \\
\text { coefficient }\end{array}$ & $P$ value \\
\hline $\operatorname{PTV}\left(\mathrm{cm}^{3}\right)$ & $578(184)$ & 0.01 & 0.92 \\
\hline $\mathrm{D}_{\max }$ & $27.2(0.3)$ & 0.13 & 0.29 \\
\hline $\mathrm{D}_{\text {mean }}$ & $26.4(0.3)$ & -0.02 & 0.87 \\
\hline \multicolumn{4}{|l|}{ Liver } \\
\hline $\mathrm{D}_{\max }$ & $26.2(2.1)$ & 0.11 & 0.39 \\
\hline $\mathrm{D}_{\text {mean }}$ & $3.2(1.4)$ & 0.23 & 0.06 \\
\hline V3 & $29(1)$ & 0.14 & 0.27 \\
\hline V5 & $27(10)$ & 0.13 & 0.29 \\
\hline V10 & $9(7)$ & 0.25 & 0.05 \\
\hline V15 & $6(4)$ & 0.17 & 0.17 \\
\hline V20 & $4(3)$ & 0.16 & 0.21 \\
\hline V25 & $2(2)$ & 0.12 & 0.36 \\
\hline \multicolumn{4}{|l|}{ Stomach } \\
\hline $\mathrm{D}_{\max }$ & $26.5(1.5)$ & 0.01 & 0.93 \\
\hline $\mathrm{D}_{\text {mean }}$ & $4.1(1.7)$ & 0.33 & 0.008 \\
\hline V3 & $30(11)$ & 0.34 & 0.005 \\
\hline V5 & $26(10)$ & 0.35 & 0.005 \\
\hline V10 & $16(7)$ & 0.31 & 0.01 \\
\hline V15 & $11(6)$ & 0.27 & 0.03 \\
\hline V20 & $7(4)$ & 0.18 & 0.15 \\
\hline V25 & $3(3)$ & 0.12 & 0.37 \\
\hline \multicolumn{4}{|l|}{ Duodenum } \\
\hline $\mathrm{D}_{\max }$ & $27.0(0.3)$ & 0.02 & 0.86 \\
\hline $\mathrm{D}_{\text {mean }}$ & $24.4(2.2)$ & 0.01 & 0.91 \\
\hline V10 & $95(8)$ & 0.01 & 0.95 \\
\hline V15 & $93(9)$ & 0.01 & 0.97 \\
\hline V20 & 89 (11) & -0.01 & 0.92 \\
\hline V25 & $79(15)$ & -0.02 & 0.88 \\
\hline \multicolumn{4}{|l|}{ Small bowel } \\
\hline $\mathrm{D}_{\max }$ & $26.7(0.6)$ & 0.16 & 0.20 \\
\hline $\mathrm{D}_{\text {mean }}$ & $5.4(2.3)$ & 0.08 & 0.49 \\
\hline V3 & $50(23)$ & 0.09 & 0.48 \\
\hline V5 & $47(22)$ & 0.09 & 0.44 \\
\hline V10 & $15(12)$ & -0.02 & 0.86 \\
\hline V15 & $10(7)$ & -0.01 & 0.94 \\
\hline V20 & $7(5)$ & 0.01 & 0.96 \\
\hline V25 & $4(4)$ & 0.07 & 0.56 \\
\hline \multicolumn{4}{|l|}{ Large bowel } \\
\hline $\mathrm{D}_{\max }$ & 24.7 (4.9) & 0.12 & 0.34 \\
\hline $\mathrm{D}_{\text {mean }}$ & $4.5(2.1)$ & 0.15 & 0.22 \\
\hline V3 & $43(16)$ & 0.19 & 0.13 \\
\hline V5 & $40(16)$ & 0.18 & 0.15 \\
\hline V10 & $12(10)$ & 0.21 & 0.08 \\
\hline V15 & $7(8)$ & 0.09 & 0.45 \\
\hline V20 & $5(6)$ & 0.09 & 0.48 \\
\hline
\end{tabular}

Table 3 (continued)

\begin{tabular}{lllc}
\hline & $\begin{array}{l}\text { Mean volume (\%) } \\
\text { or dose (GyE) with } \\
\text { SD }(n=47)\end{array}$ & $\begin{array}{l}\text { Kendall's correlation } \\
\text { coefficient }\end{array}$ & $P$ value \\
\hline V25 & $2(3)$ & 0.09 & 0.50 \\
\hline
\end{tabular}

Significant dosimetric parameters are highlighted in bold

$S D$ standard deviation

or both, and those who did not, although our small sample could have limited our power to detect a difference. Other larger, radiotherapy-based studies have also failed to identify younger age, gender, or alcohol intake - covariates significant in chemotherapy-based studies [13] — as significant predictors for nausea and vomiting during radiotherapy $[2,3]$. These observations suggest the possibility of different underlying patient risk factors between chemotherapy and radiotherapy-induced nausea and vomiting, but further confirmatory studies are warranted.

Despite the small numbers in our cohort, our study has several strengths. In this single-institution prospective study, all patients were rigorously staged and confirmed to have resectable pancreatic cancer, evaluated twice weekly by the treating team per study recommendations, received the same chemoradiotherapy treatment, and had contours performed by a single, experienced, anatomist and dosimetrist. Thus, these conclusions are derived from a relatively homogeneous sample. To our knowledge, this is the first study evaluating dosimetric correlates for nausea and vomiting among patients receiving hypofractionated radiotherapy to the pancreas. Hypofractionated regimens have been increasingly used to treat pancreatic cancer given both its short treatment duration for patients with otherwise limited life-spans and tolerable acute toxicity profile [6-8]. Although our findings require validation, they may in the future be used to identify patients who are at higher risk of treatment-induced nausea and vomiting and who might benefit from prophylactic antiemetic medications.

A few points, however, require further consideration. The correlation coefficients for each of our five dosimetric predictors suggest a weak to moderate correlation and may explain only a small proportion of the nausea, vomiting, or both observed during treatment. This is consistent with the hypothesis that the mechanisms underlying treatment-induced nausea and vomiting are complex and likely multifactorial. On the other hand, the weak to moderate correlation may be secondary to our chosen endpoint: physician-reported nausea, vomiting, or both. Although commonly used, physician-reported nausea and vomiting was ascertained after aggressive, prophylactic use of ondansetron or prochlorperazine and likely captured fewer events. Despite this limitation, we were still able to identify significant dosimetric parameters. Physician-reported data is generally thought to underreport toxicity, although one study suggests that there is generally good agreement between patients and physicians on the presence of nausea symptoms 
(kappa coefficient, $88.7 \%$ ) but less agreement on the severity (74 \%) [19]. Nonetheless, although no significant difference was detected in our cohort, patients who experienced treatment-related nausea and vomiting also more frequently required rescue antiemetic medication during treatment compared with patients who did not have nausea or vomiting (73 versus $55 \% ; p=0.34$ ). Our analysis may be underpowered to detect a difference, and further studies are warranted to confirm whether these surrogate endpoints may be correlated. Future studies to confirm our results ideally should use patientreported toxicity through diaries or questionnaires.

Our patients were treated with hypofractionated radiotherapy (5 GyE per fraction), and therefore, our results may not be generalizable to patients who receive conventionally fractionated treatment. However, the frequency of grades 1 and 2 nausea and vomiting in our study was similar to that reported in other retrospective studies employing capecitabine and conventionally fractionated radiotherapy $[10,11]$. It is unclear whether dose per fraction is a risk factor for treatment-induced nausea and vomiting. One multicenter study observed that among 914 patients, dose per fraction (less than 3 Gy versus greater or equal to $3 \mathrm{~Gy}$ ) was not a significant risk factor for radiation-induced nausea and vomiting, although only 42 of these patients received radiotherapy to the upper abdomen [3]. As stereotactic body radiotherapy (SBRT) is increasingly being used to treat upper gastrointestinal tumors, these results may be extrapolated for SBRT treatments. One caveat is that in our study, limited elective nodal treatment was included, and therefore, the treated target volumes may be larger than those used for SBRT.

Additionally, while we found a correlation with nausea or vomiting and stomach dose, this analysis is hampered by interand intra-fractional variability in both the stomach's position and filling at any given time. Because the stomach was contoured on a single CT dataset, we did not assess to what degree the stomach may have been distended on a daily basis. Thus, it is not possible to know what dose the stomach actually received. Other studies have documented that the stomach not only moves but also is subject to a significant amount of deformations [20]. However, contouring the stomach likely represents a reasonable surrogate for stomach dose, insofar as the dose is kept out of the left upper quadrant intentionally.

Radiotherapy was delivered concurrently with capecitabine, a moderately emetogenic drug that likely contributes to treatment-induced nausea and vomiting. However, since all patients in our study received a fixed capecitabine dose based on their body surface area, the emetogenic effect of capecitabine may be consistent among our cohort. Furthermore, use of concurrent emetogenic chemotherapy and radiotherapy is increasingly becoming the standard of care for definitive or adjuvant treatment of many upper abdominal sites [21, 22].

It is unclear whether the dosimetric predictors or physicianreported nausea and vomiting are correlated with late gastric complications such as delayed gastric emptying or pathological findings of radiation-induced mucosal changes. In our study, none of the patients who experienced nausea or vomiting had late gastric complications (median follow-up time of 238 days from end of radiation; range, 7-1,523). Two $(8 \%)$ of these patients who underwent a Whipple had mucosal changes consistent with radiation effect, compared with $5 \%$ of patients who had no nausea or vomiting and underwent a Whipple. However, given the retrospective nature of our study, it is difficult to interpret these findings. Finally, our results are hypothesis generating and require further validation in other larger cohorts.

\section{Conclusion}

Hypofractionated radiation to the pancreas concurrent with capecitabine was well-tolerated with $51 \%$ and $4 \%$ of patients experiencing grades 1 and 2 nausea and vomiting, respectively. Hypofractionated radiotherapy to the stomach $\left(\mathrm{D}_{\text {mean }}, \mathrm{V} 3, \mathrm{~V} 5\right.$, V10, V15) was correlated with presence of nausea, vomiting, or both during treatment with stomach V10 $\geq 11.5 \%$ as the best predictor. While these findings require validation, they suggest that dosimetric constraints could be considered in order to minimize the risk of treatment-induced nausea and vomiting and help identify high-risk patients who may benefit from prophylactic antiemetic medications.

Conflict of interest Yolanda Tseng, Jennifer Wo, Marek Ancukiewicz, Judith Adams, Nicolas Depauw, Harvey Mamon, and Theodore Hong declare that they have no conflict of interest.

Statements on human studies All human and animal studies were approved by the appropriate ethics committee and were therefore been performed in accordance with the ethical standards laid down in the 1964 Declaration of Helsinki and its later amendments. All persons gave their informed consent prior to their inclusion in the study. This study included patients from phase I/II prospective trial, which followed the ethical standard of our governing body.

\section{References}

1. Feyer PC, Stewart AL, Titlbach OJ (1998) Aetiology and prevention of emesis induced by radiotherapy. Support Care Cancer 6:253-260

2. Maranzano E, De Angelis V, Pergolizzi S et al (2010) A prospective observational trial on emesis in radiotherapy: analysis of 1020 patients recruited in 45 Italian radiation oncology centres. Radiother Oncol 94:36-41

3. IGARR (1999) Radiation-induced emesis: a prospective observational multicenter Italian trial. Int J Radiat Oncol Biol Phys 44:619-625

4. Court Brown WM (1953) Symptomatic disturbance after single therapeutic dose of $\mathrm{X}$ rays: its relationship to the general radiation syndrome. Br Med J1:802-805

5. Danjox CE, Rider WD, Fitzpatrick PJ (1979) The acute radiation syndrome. A memorial to William Michael Court-Brown. Clin Radiol 30:581-584 
6. Mahadevan A, Miksad R, Goldstein M et al (2011) Induction gemcitabine and stereotactic body radiotherapy for locally advanced nonmetastatic pancreas cancer. Int J Radiat Oncol Biol Phys 81: e615-e622

7. Chang DT, Schellenberg D, Shen J et al (2009) Stereotactic radiotherapy for unresectable adenocarcinoma of the pancreas. Cancer 115:665-672

8. Goyal K, Einstein D, Ibarra RA et al (2012) Stereotactic body radiation therapy for nonresectable tumors of the pancreas. J Surg Res 174:319-325

9. Hong TS, Ryan DP, Blaszkowsky LS et al (2011) Phase I study of preoperative short-course chemoradiation with proton beam therapy and capecitabine for resectable pancreatic ductal adenocarcinoma of the head. Int J Radiat Oncol Biol Phys 79:151-157

10. Ben-Josef E, Shields AF, Vaishampayan U et al (2004) Intensitymodulated radiotherapy (IMRT) and concurrent capecitabine for pancreatic cancer. Int J Radiat Oncol Biol Phys 59:454-459

11. Das P, Wolff R, Abbruzzese J et al (2006) Concurrent capecitabine and upper abdominal radiation therapy is well tolerated. Radiat Oncol $1: 41$

12. Saif MW, Eloubeidi MA, Russo S et al (2005) Phase I study of capecitabine with concomitant radiotherapy for patients with locally advanced pancreatic cancer: expression analysis of genes related to outcome. J Clin Oncol 23:8679-8687

13. Pollera CF, Giannarelli D (1989) Prognostic factors influencing cisplatin-induced emesis. Definition and validation of a predictive logistic model. Cancer 64:1117-1122

14. Lee VHF, Ng SCY, Leung TW et al (2012) Dosimetric predictors of radiation-induced acute nausea and vomiting in IMRT for nasopharyngeal cancer. Int J Radiat Oncol Biol Phys 84:176-182
15. Nakamura A, Shibuya K, Matsuo Y et al (2012) Analysis of dosimetric parameters associated with acute gastrointestinal toxicity and upper gastrointestinal bleeding in locally advanced pancreatic cancer patients treated with gemcitabine-based concurrent chemoradiotherapy. Int J Radiat Oncol Biol Phys 84:369-375

16. Kozak KR, Kachnic LA, Adams J et al (2007) Dosimetric feasibility of hypofractionated proton radiotherapy for neoadjuvant pancreatic cancer treatment. Int J Radiat Oncol Biol Phys 68:1557-1566

17. Bouchard M, Amos RA, Briere TM et al (2009) Dose escalation with proton or photon radiation treatment for pancreatic cancer. Radiother Oncol 92:238-243

18. Yovino S, Poppe M, Jabbour S et al (2011) Intensity-modulated radiation therapy significantly improves acute gastrointestinal toxicity in pancreatic and ampullary cancers. Int J Radiat Oncol Biol Phys 79:158-162

19. Cirillo M, Venturini M, Ciccarelli L et al (2009) Clinician versus nurse symptom reporting using the National Cancer Institute- common terminology criteria for adverse events during chemotherapy: results of a comparison based on patient's self-reported questionnaire. Ann Oncol 20:1929-1935

20. Watanabe $M$, Isobe $K$, Takisima $H$ et al (2008) Intrafractional gastric motion and interfractional stomach deformity during radiation therapy. Radiother Oncol 87:425-431

21. Cooper JS, Guo MD, Herskovic A et al (1999) Chemoradiotherapy of locally advanced esophageal cancer: long-term follow-up of a prospective randomized trial (RTOG 85-01). JAMA 281:1623-1627

22. Smalley SR, Benedetti JK, Haller DG et al (2012) Updated analysis of SWOG-directed intergroup study 0116: a phase III trial of adjuvant radiochemotherapy versus observation after curative gastric cancer resection. J Clin Oncol 30:2327-2333 